\title{
Sequential virus monitoring of pediatric patients with hematopoietic stem cell transplantation by multiplex PCR method
}

\author{
Masayuki Nagasawa ${ }^{1,2 *}$, Noriko Mitsuiki ${ }^{1}$, Akifumi Endo ${ }^{1}$, Yuki Aoki ${ }^{1}$, Toshiaki Ono ${ }^{1}$, Takeshi Isoda ${ }^{1}$, Masatoshi Takagi ${ }^{1}$, Michiko \\ Kajiwara $^{1}$, Norio Shimizu ${ }^{3}$ and Tomohiro Morio ${ }^{1}$ \\ ${ }^{1}$ Department of Pediatrics and Developmental Biology. Tokyo Medical and Dental University, Tokyo, Japan \\ ${ }^{2}$ Department of Pediatrics, Musashino Red Cross Hospital, Tokyo, Japan \\ ${ }^{3}$ Department of Virology, Tokyo Medical and Dental University, Tokyo, Japan
}

\begin{abstract}
Aim: To investigate the risk factors of virus infection and clinical significance of virus monitoring in pediatric patients with allogeneic stem cell transplantations (SCT).

Methods: Peripheral blood was weekly checked for HSV-1, 2, VZV, EBV, CMV, HHV-6,-7,-8, BKV, JCV and PBV19 by multiplex PCR method until day100120 in 33 pediatric patients with 35 allogeneic SCT performed between 2004 and 2009. Occasionally, ADV was examined in cases with suspected clinical symptoms. Transplantation-related mortality (TRM) was compared with that of 35 SCT between 1998 and 2004 when virus monitoring was not introduced.

Result: EBV was positive for 17cases (48.6\%), CMV for 10 (28.6\%) and HHV-6 for 13 (37.1\%). Mean time of onset was 30th day for HHV-6, 41st day for CMV, and 46th day for EBV. HSV-1 was positive for three cases, BKV and JCV for two cases, and HSV-2, VZV, HHV-7 and ADV for one case. No virus was detected in 12 cases (34.3\%). Multivariate analysis showed that ATG and HHV-6 infection were statistically relevant (odds ratio 40.03, p=0.04). GvHD III-IV was related with CMV (odds ratio 6.25, $\mathrm{p}=0.09$ ) and $\mathrm{EBV}$ (odds ratio 10.47, $\mathrm{p}=0.08$ ) infection, and reduced-intensity conditioning (RIC) was related with HHV-6 infection (odds ratio 8.65, $\mathrm{p}=0.16$ ), although not statistically significant. To detect CMV infection, PCR was more sensitive than CMV pp65 antigenemia. Positive PCR result for CMV, EBV, or HHV-6 before SCT seemed to be a risk factor for reactivation of each virus. Positive antibody for CMV of the recipient was also a risk factor of CMV infection. TRM in 2004-2009 cohort was 0.208 and TRM in 1998-2004 cohort was 0.301, and the introduction of virus monitoring improved TRM, although not statistically significant.
\end{abstract}

Conclusion: Multiplex virus monitoring before and after SCT is possibly useful for the management of pediatric patients with SCT. Further examination is necessary to confirm its clinical significance.

\begin{abstract}
Abbreviations: SCT: Stem Cell Transplantation; PCR: Polymerase Chain Reaction; TRM: Transplantation-Related Mortality; EBV: Epstein-Barr Virus; CMV: Cytomegalovirus; VZV: Varicella Zoster Virus; HHV-6: Human Herpesvirus-6; HHV-7: Human Herpesvirus-7; HHV-8: Human Herpesvirus-8; PVB19: Parvovirus B19; MAC: Myeloablative Conditioning; RIC: Reduced-Intensity Conditioning; GvHD: Graft versus Host Disease
\end{abstract}

\section{Introduction}

Viral infection is still a main factor of the morbidity and mortality after the hematopoietic stem cell transplantation (SCT) while supportive care has been strengthened [1]. Furthermore, with the expansion of the transplant feasibility, the importance of opportunistic infection increases along with the development of novel immunosuppressant and conditioning regimen [2]. Prophylactic treatment reduced the incidence of early viral infection, but increased the late viral infection by compromising the anti-viral immune recovery, resulting in no improvement of overall survival [3,4]. Recently, it has been reported that early detection of targeted viruses and preemptive treatment are important to have good outcomes [5]. There are various reports in the adult SCT, but there are few examinations about the infection after pediatric SCT. From the results of viral monitoring in our institute, we investigate risk factors of virus infection after the pediatric SCT and examined the clinical impact of sequential virus monitoring by comparing with the historical control in which virus monitoring was not introduced.

\section{Methods}

Subject: For 35 hematopoietic stem cell transplantation cases performed in Tokyo Medical and Dental University between September 2004 and December 2009 (Table 1), we performed a sequential virus monitoring using the multiplex PCR method with a peripheral blood specimen once a week until 100-120 days after transplant. In 31 cases,

Correspondence to: Masayuki Nagasawa, Department of Pediatrics, Musashino Red Cross Hospital 1-26-1, Kyonan-cho, Musashino-city, Tokyo 180-8610, Japan; Tel +81-422-32-3111; Fax +81-422-32-3525; E-mail: mnagasawa.ped@tmd.ac.jp

Key words: stem cell transplantation, pediatric SCT, multiplex PCR, CMV, EBV, HHV-6

Received: July 12, 2016; Accepted: August 22, 2016; Published: August 25, 2016 
Table 1. Clinical profiles of the patients with SCT (2004-2009) under virus monitoring.

\begin{tabular}{|c|c|c|c|c|c|c|c|c|c|c|c|}
\hline Patient & Disease & $\begin{array}{c}\text { Age } \\
\text { (yaer) }\end{array}$ & $\begin{array}{l}\text { Donor } \\
\text { origin }\end{array}$ & $\begin{array}{c}H<A \\
\text { (phenotype) }\end{array}$ & $\begin{array}{l}\text { Conditioning } \\
\text { regimen }\end{array}$ & GVHD prophylaxis & $\begin{array}{l}\text { Acute } \\
\text { GVHD }\end{array}$ & Toxicity/complication & $\begin{array}{c}\text { Engraftmen } \\
\text { t(day) }\end{array}$ & $\begin{array}{l}\text { Outcome } \\
\text { (day) }\end{array}$ & Positive virus \\
\hline 1 & CML & 19 & UR-BM & 6 & $\mathrm{TBI}+\mathrm{CY}$ & TAC+SMTX & IV & TMA & 16 & dead (D460) & $\mathrm{EBV} \cdot \mathrm{CMV}$ \\
\hline 2 & WAS & 1 & URCB & 5 & $\mathrm{BU}+\mathrm{CY}+\mathrm{ATG}$ & $\mathrm{CSP}+\mathrm{sMTX}+\mathrm{mPSL}$ & 0 & - & 19 & alive & - \\
\hline 3 & $\mathrm{CID}$ & 1 & R-BM & 5 & Flu+L-PAM+ATG & TAC+SMTX & III & encephalopathy & 12 & alive & $\mathrm{EBV} \cdot \mathrm{HHV} \cdot \mathrm{HSV}_{1} \cdot \mathrm{HSV}_{2}$ \\
\hline 4 & ALL & 5 & R-BM & 6 & $\mathrm{BU}+\mathrm{CY}+\mathrm{VP}$ & CSP+SMTX & 0 & - & 14 & alive & $\begin{array}{c}\mathrm{CMV} \cdot \mathrm{HSV} 1 \cdot \mathrm{HSV} 2 \cdot \mathrm{EBV} \cdot \\
\mathrm{HHV} 6\end{array}$ \\
\hline 5 & AML & 14 & UR-BM & 6 & $\mathrm{BU}+\mathrm{CY}+\mathrm{L}-\mathrm{PAM}$ & TAC+sMTX & II & ES & 15 & alive & $\mathrm{CMV} \cdot \mathrm{EBV}$ \\
\hline 6 & AML-Down & 1 & URCB & 5 & $B U+L-P A M+C A$ & $\mathrm{CSP}+\mathrm{sMTX}$ & III & hemorrhage(lung,brain) & - & dead (D125) & $\mathrm{CMV} \cdot \mathrm{EBV}$ \\
\hline 7 & AML/MDSRAEB & 14 & UR-BM & 6 & $\mathrm{BU}+\mathrm{CY}+\mathrm{L}-\mathrm{PAM}$ & CSP+SMTX & IIII & TMA $\cdot E S$ & 21 & alive & $\mathrm{CMV} \cdot \mathrm{HSV} 1 \cdot \mathrm{EBV}$ \\
\hline 8 & ALL & 0 & URCB & 5 & $\mathrm{BU}+\mathrm{CY}+\mathrm{VP}$ & $\mathrm{CSP}+\mathrm{SMTX}$ & 1 & ES & 16 & alive & $\mathrm{HHV6} \cdot \mathrm{HSV} 1 \cdot \mathrm{VZV} \cdot \mathrm{EBV}$ \\
\hline 9 & ALD & 12 & URCB & 4 & TLI+L-PAM+ATG & CSP+PSL & 0 & - & 13 & alive & $\mathrm{EBV} \cdot \mathrm{CMV} \cdot \mathrm{HHV6}$ \\
\hline 10 & WAS & 21 & R-BM & 5 & $\mathrm{BU}+\mathrm{CY}+\mathrm{ATG}$ & TAC+sMTX & III & TMA $\cdot E S$ & 19 & alive & $\mathrm{EBV} \cdot \mathrm{CMV} \cdot \mathrm{BKV} \cdot \mathrm{JCV}$ \\
\hline 11 & AML & 13 & R-PBSC & 5 & $\mathrm{TBI}+\mathrm{CY}$ & TAC+SMTX & III & ES & 15 & dead (D374) & HHV6 $\cdot$ EBV \\
\hline 12 & CAEBV & 16 & UR-BM & 6 & Flu+L-PAM+ATG & TAC+SMTX & IV & $T M A \cdot E S$ & 15 & dead (D59) & $\mathrm{CMV} \cdot \mathrm{HHV6} \cdot \mathrm{JCV} \cdot \mathrm{EBV}$ \\
\hline 13 & AML & 4 & UR-BM & 6 & $\mathrm{TBI}+\mathrm{CY}$ & TAC+SMTX & 1 & - & 22 & alive & $\mathrm{EBV} \cdot \mathrm{CMV}$ \\
\hline 14 & AML & 17 & R-BM & 6 & $\mathrm{TBI}+\mathrm{CY}$ & CSP+sMTX & I & - & 16 & alive & - \\
\hline 15 & ALL & 4 & R-BM & 6 & $\mathrm{TBI}+\mathrm{CY}+\mathrm{VP}$ & CSP+SMTX & 0 & - & 16 & relapse $(\rightarrow \# 20)$ & - \\
\hline 16 & LAD & 0 & UR-BM & 6 & $\mathrm{Flu}+\mathrm{CY}+2 \mathrm{~Gy}$ & TAC+sMTX & II & VOD & 12 & rejection $(\rightarrow \# 18)$ & EBV \\
\hline 17 & ALL & 13 & R-BM & 6 & $\mathrm{TBI}+\mathrm{CY}+\mathrm{VP}$ & $\mathrm{CSP}+\mathrm{SMTX}$ & II & - & 19 & alive & - \\
\hline 18 & $\operatorname{LAD}(2 n d)$ & 1 & UR-BM & 5 & $\mathrm{iBU}+\mathrm{CY}$ & TAC+sMTX & II & - & 14 & alive & - \\
\hline 19 & ALL & 14 & UR-BM & 6 & $\mathrm{TBI}+\mathrm{CY}+\mathrm{VP}$ & TAC+SMTX & II & ES & 12 & dead (D60) & $\mathrm{EBV} \cdot \mathrm{HHV} 6$ \\
\hline 20 & ALL (2nd) & 4 & UR-BM & 6 & $\mathrm{iBU}+\mathrm{CY}+\mathrm{L}-\mathrm{PAM}$ & TAC+SMTX & 0 & VOD & 19 & dead (D60) & HHV6 $\cdot A D V$ \\
\hline 21 & ALL & 11 & UR-BM & 6 & $\mathrm{TBI}+\mathrm{CY}+\mathrm{VP}$ & TAC+sMTX & II & intestial pneumonia & 17 & alive & HHV6 $\cdot \mathrm{BKV} \cdot \mathrm{EBV}$ \\
\hline 22 & AML & 16 & UR-BM & 6 & Flu+L-PAM+2Gy & TAC+SMTX & II & TMA & 22 & alive & HHV6 \\
\hline 23 & WAS & 0 & R-BM & 6 & $\mathrm{iBU}+\mathrm{CY}$ & CSP+sMTX & 0 & ES & 16 & alive & - \\
\hline 24 & SCID & 0 & URCB & 6 & Flu+L-PAM & $\mathrm{CSP}+\mathrm{PSL}$ & III & $\begin{array}{c}\text { TMA - Liver dysfunction * } \\
\text { ES }\end{array}$ & 14 & $\begin{array}{c}\text { rejection - dead } \\
\text { (D491) }\end{array}$ & - \\
\hline 25 & AML & 11 & URCB & 4 & $\mathrm{TBI}+\mathrm{CY}+\mathrm{ATG}$ & TAC+SMTX & I & - & 24 & alive & HHV6 \\
\hline 26 & WAS & 0 & R-BM & 6 & $\mathrm{iBU}+\mathrm{CY}$ & CSP+SMTX & 0 & - & 12 & alive & - \\
\hline 27 & ALL & 0 & URCB & 5 & $i B U+C Y+V P$ & CSP+SMTX & II & $\mathrm{VOD} \cdot \mathrm{ES}$ & 22 & alive & - \\
\hline 28 & SCID & 0 & URCB & 5 & Flu+L-PAM & $T A C+M T X+m P S L$ & 0 & TMA - HPS - ES & - & dead (D44) & HHV6 \\
\hline 29 & SCID & 0 & URCB & 6 & Flu+L-PAM & TAC+SMTX & I & ES & 17 & alive & - \\
\hline 30 & XHIM & 17 & UR-BM & 6 & Flu+iBU+CY & TAC+SMTX & I & ES & 17 & alive & EBV \\
\hline 31 & SCID & 0 & URCB & 6 & Flu+L-PAM & $T A C+s M T X+m P S L$ & III & ES & 21 & alive & - \\
\hline 32 & SCID & 1 & URCB & 6 & Flu+L-PAM & TAC+SMTX & II & BCG reactivation - ES & 18 & alive & - \\
\hline 33 & AML & 1 & R-BM & 6 & $\mathrm{iBU}+\mathrm{L}-\mathrm{PAM}$ & CSP & IV & $\mathrm{BO} \cdot \mathrm{ES}$ & 9 & alive & HHV7 \\
\hline 34 & $\overline{A M L}$ & 13 & UR-BM & 6 & $\mathrm{TBI}+\mathrm{CY}$ & TAC+sMTX & II & ES & 21 & alive & $\mathrm{CMV} \cdot \mathrm{HHV6}$ \\
\hline 35 & $\mathrm{DC}$ & 9 & UR-BM & 6 & Flu+L-PAM+ATG & TAC+SMTX & III & encephalopathy - ES & 12 & alive & EBV \\
\hline
\end{tabular}

PCR analysis was performed since one month before SCT. We analyzed urine, stool, intestinal mucosa specimen when suspected symptoms were observed.

As a historical control, 35 patients who were transplanted between 1998 and 2004 (Table 2), when virus monitoring was not introduced at our institute were analyzed.

Laboratory procedure: We analyzed herpes simplex virus type 1 and 2 (HSV-1, -2), varicella zoster virus (VZV), Epstein-Barr virus (EBV), cytomegalovirus (CMV), human herpesvirus 6, 7 and 8 (HHV6,-7,-8), BK virus (BKV), JC virus (JCV), Parvovirus B19 (PVB19). Adeno virus (ADV) was analyzed when suspected. After nucleic acid extraction from whole blood, multiplex PCR was performed and then hybridization to each virus was carried out. We detect each virus by a melting curve analysis qualitatively.

In qualitatively positive cases, real time PCR for each virus was performed, respectively. We defined that virus was positive when virus was detected in real time PCR method as described before [6-8]. PCR assay was performed at Cell Therapy Center of Tokyo Medical and Dental University Hospital. Primers used in multiplex PCR and quantitative nested PCR for virus detection are listed in Table 3.

Procedure of hematopoietic stem cell transplantation and supportive care: In principle, GvHD prophylaxis consisted of cyclosporine (CSP) plus short-term methotrexate (MTX) for human leukocyte antigen (HLA)-identical-related donors, and tacrolimus (TAC) plus short-term MTX for HLA-identical unrelated and HLAmismatched-related donors. For unrelated umbilical cord blood donors, CSP or TAC and short-term MTX were used. In some cases, methylprednisolone (mPSL) or prednisolone (PSL) was added as GvHD prophylaxis. CSP was administered twice daily, and blood trough level was adjusted between $150-250 \mathrm{ng} / \mathrm{mL}$, and TAC was continuously administered and blood level was maintained between $8-12 \mathrm{ng} / \mathrm{mL}$.

As a prophylaxis for infections, aciclovir and anti-fungal drug (fluconazole or micafungin sodium) were started at 7 days and one day before SCT, respectively. Trimethoprim-sulfamethoxazole (ST combination) was discontinued one day before SCT, and restarted after engraftment. In a case with previous Pneumocystis jirovecii infection, ST combination was continued during SCT. Immunoglobulin was administered to keep serum IgG level more than $500 \mathrm{mg} / \mathrm{dL}$ every 2 weeks. We preemptively started ganciclovir or foscarnet sodium hydrate for clinically suspected CMV infection case or case with viral load of $1 \times 10^{2}$ copies/ $\mu \mathrm{gDNA}$ and more with positive CMV pp65 antigenemia (more than 5 positive cells out of 50,000 cells). Engraftment was determined as the first day when neutrophil count exceeded $500 / \mu l$ for three consecutive days. 
Table 2. Clinical profiles of the patients with SCT (1998-2004) without virus monitoring.

\begin{tabular}{|c|c|c|c|c|c|c|c|c|c|}
\hline Patient & Disease & $\begin{array}{c}\text { Age } \\
\text { (yaer) }\end{array}$ & Donor origin & \begin{tabular}{|c|} 
HLA \\
(phenotype)
\end{tabular} & Conditioning regimen & GVHD prophylaxis & aGVHD & Toxicity/complication & $\begin{array}{c}\begin{array}{c}\text { Outcome } \\
\text { (day) }\end{array} \\
\end{array}$ \\
\hline 1 & ALL & 17 & UR-BM & 6 & $\mathrm{TBI}+\mathrm{CY}+\mathrm{VP}$ & CSP+SMTX & 1 & & alive \\
\hline 2 & HIM & 17 & R-PBSC & 6 & $\mathrm{TBI}+\mathrm{CY}$ & $\mathrm{CSP}+\mathrm{mPSL}$ & 0 & TMA, VOD, P.aeruginosa & Dead(D69) \\
\hline 3 & AML & 3 & R-BM & 6 & $\mathrm{TBI}+\mathrm{CY}+\mathrm{VP}$ & CSP+sMTX & 1 & & Dead(D449) \\
\hline 4 & SCID & 0 & URCB & 5 & $\mathrm{BU}+\mathrm{CY}$ & CSP+sMTX & 0 & & alive \\
\hline 5 & HIM & 3 & R-BM & 6 & $\mathrm{BU}+\mathrm{CY}$ & CsSP+sMTX & 1 & & alive \\
\hline 6 & $\mathrm{ALL}$ & 2 & URCB & 5 & $\mathrm{TBI}+\mathrm{CY}+\mathrm{VP}$ & CSP & II & & alive \\
\hline 7 & HIM & 14 & R-BM & 6 & $\mathrm{BU}+\mathrm{CY}$ & CSP+sMTX & IV & Aspergillosis & Dead(D89) \\
\hline 8 & WAS & 19 & $\mathrm{R}-\mathrm{BM}$ & 6 & $\mathrm{BU}+\mathrm{CY}$ & $\mathrm{CSP}+\mathrm{SMTX}+\mathrm{PSL}$ & 0 & & alive \\
\hline 9 & WAS & 1 & UR-BM & 6 & $\mathrm{BU}+\mathrm{CY}+\mathrm{ATG}$ & CSP+sMTX+ATG & II & & alive \\
\hline 10 & HIM & 3 & UR-BM & 6 & $\mathrm{BU}+\mathrm{CY}$ & CSP+SMTX & II & & alive \\
\hline 11 & HIM & 17 & R-BM & 6 & $\mathrm{BU}+\mathrm{CY}$ & CSP+sMTX & II & & alive \\
\hline 12 & AML & 9 & R-BM & 6 & $\mathrm{TBI}+\mathrm{CY}+\mathrm{VP}$ & CSP+sMTX & 0 & & alive \\
\hline 13 & CAEBV & 18 & UR-BM & 6 & $\mathrm{TBI}+\mathrm{CY}+\mathrm{VP}$ & CSP+sMTX & 1 & $\mathrm{BO}$ & Dead(D609) \\
\hline 14 & AML & 10 & URCB & 4 & BU+LPAM & CSP & II & & Dead(D631) \\
\hline 15 & CAEBV & 14 & R-PBSC & 4 & $\mathrm{TBI}+\mathrm{CY}+\mathrm{VP}$ & TAC & III & P.jirovecii, GI-bleeding & Dead(D202) \\
\hline 16 & $\mathrm{HIM}$ & 3 & UR-BM & 6 & $\mathrm{BU}+\mathrm{CY}$ & CSP+sMTX & II & & alive \\
\hline 17 & ALL & 16 & UR-BM & 6 & $\mathrm{TBI}+\mathrm{CY}+\mathrm{VP}$ & TAC+MTX & 1 & & alive \\
\hline 18 & ALL & 4 & UR-BM & 6 & $\mathrm{TBI}+\mathrm{CY}+\mathrm{VP}$ & TAC+sMTX & 0 & & alive \\
\hline 19 & WAS & 0 & UR-BM & 6 & $\mathrm{BU}+\mathrm{CY}+\mathrm{ATG}$ & TAC+sMTX & 0 & BO & alive \\
\hline 20 & WAS & 11 & UR-BM & 6 & $\mathrm{BU}+\mathrm{CY}+\mathrm{ATG}$ & TAC+SMTX & IV & $\begin{array}{c}\text { BO, encephalopathy } \\
\text { P.aeruginosa }\end{array}$ & Dead(D373) \\
\hline 21 & SCID & 0 & URCB & 5 & $\mathrm{BU}+\mathrm{CY}$ & CSP+sMTX & 0 & VOD & Dead(D5) \\
\hline 22 & WAS & 1 & UR-BM & 6 & $\mathrm{BU}+\mathrm{CY}+\mathrm{ATG}$ & $\mathrm{TAC}+\mathrm{sMTX}+\mathrm{mPSL}$ & 0 & & alive \\
\hline 23 & HIM & 19 & $\mathrm{R}-\mathrm{BM}$ & 6 & $\mathrm{BU}+\mathrm{CY}$ & $\mathrm{CSP}+\mathrm{sMTX}$ & 0 & & alive \\
\hline 24 & CAEBV & 21 & R-PBSC & 5 & Flu+LPAM+ATG & TAC+sMTX & III & EBV-LPD & Dead(D417) \\
\hline 25 & HPS & 1 & URCB & 5 & $\mathrm{BU}+\mathrm{CY}+\mathrm{VP}$ & $\mathrm{CSP}+\mathrm{mPSL}$ & 0 & & alive \\
\hline 26 & $\mathrm{OP}$ & 0 & URCB & 6 & Flu+LPAM+ATG & CSP+SMTX+PSL & III & VOD & alive \\
\hline 27 & HPS & 1 & URCB & 5 & none & $\mathrm{CSP}+\mathrm{mPSL}$ & 0 & & alive \\
\hline 28 & ALL & 1 & R-BM & 5 & $\mathrm{TBI}+\mathrm{CY}+\mathrm{VP}$ & TAC+sMTX & II & cGVHD & alive \\
\hline 29 & WAS & 1 & URCB & 5 & $\mathrm{BU}+\mathrm{CY}+\mathrm{ATG}$ & $\mathrm{CSP}+\mathrm{SMTX}+\mathrm{PSL}$ & III & TMA, CMV enteritis & Dead(D216) \\
\hline 30 & CAEBV & 22 & R-BM & 6 & Flu+LPAM & CSP+sMTX & 1 & & alive \\
\hline 31 & CID & 3 & URCB & 6 & Flu+LPAM+ATG & $\mathrm{CSP}+\mathrm{PSL}$ & 0 & VOD, ARF & Dead(D68) \\
\hline 32 & ALL & 13 & UR-BM & 6 & $\mathrm{TBI}+\mathrm{CY}+\mathrm{VP}$ & TAC+sMTX & 0 & & alive \\
\hline 33 & CID & 10 & URCB & 5 & Flu+LPAM+ATG & TAC+sMTX & III & cGVHD & Dead(D590) \\
\hline 34 & SCID & 0 & URCB & 6 & Flu+LPAM+ATG & $\mathrm{CSP}+\mathrm{mPSL}$ & 0 & & alive \\
\hline 35 & AML & 4 & URCB & 5 & TBI+LPAM & CSP & 0 & ARDS & Dead(D17) \\
\hline
\end{tabular}

Statistics: We calculated controlled odds ratio by the Chi-squared test and logistic regression assay (95\% confidence interval). A P-value of less than 0.05 was considered statistically significant difference.

\section{Results}

Transplantation background (Table 1): 35 hematopoietic stem cell transplantations in 33 patients were included (Table 1). Two patients received $2^{\text {nd }}$ SCT. Average age of patients was 7.2 years (from 7 months to 21 years) and median age was 4 years. Nine patients received related bone marrow (R-BM), 14 received unrelated bone marrow (UR-BM), 11 received unrelated umbilical unrelated cord blood (URCB), and one received related peripheral blood stem cells (R-PBSC). HLA were genetically matched (HLA-A, B, DRB1) in 24 cases, one locus mismatched in 9 cases, and two locus mismatched in two cases. 23 patients received myeloablative conditioning (MAC) and 12 had reduced-intensity conditioning (RIC). Conditioning regimens included total-body irradiation (TBI 12Gy) + cyclophosphamide (CY) \pm etoposide (VP16) \pm anti-thymocyte globulin (ATG) of ten cases, total nodal irradiation (TLI 12Gy)+melphalan (L-PAM)+ATG of one case, busulfan $(\mathrm{BU})+\mathrm{CY} \pm \mathrm{VP} 16 \pm \mathrm{L}-\mathrm{PAM} \pm \mathrm{ATG}$ of 11 cases, $\mathrm{BU}+\mathrm{CY}+$ fludarabine $(\mathrm{Flu})$ of one, $\mathrm{BU}+\mathrm{L}-\mathrm{PAM} \pm$ cytarabine $(\mathrm{CA})$ of two, Flu+CY+TBI $2 \mathrm{~Gy}$ of one, and Flu+L-PAM \pm ATG of 9 cases.
GvHD prophylaxis included CSP of one case, CSP + PSL of two cases, CSP + sMTX of 10 cases, CSP + sMTX + mPSL of one case, TAC+sMTX of 19 cases and TAC+sMTX + mPSL of two cases.

The patients were; seven acute lymphocytic leukemia (ALL), ten acute myeloid leukemia (AML), one chronic myelogenous leukemia (CML), one chronic active EB virus infection (CAEBV), four WiskottAldrich syndrome (WAS), five severe combined immunodeficiency (SCID), one hyper IgM syndrome (XHIM), one leukocytes adhesion deficiency (LAD), one $\mathrm{T}$ cells immunodeficiency (CID), one dyskeratosis congenita (DC), and one adrenoleukodystrophy (ALD).

Transplant results (Table 1): Mean engraftment day was 16.6 days (9-24 days and median of 16 days), except for two cases without engraftment. Engraftment syndrome (ES) according to the diagnostic criteria by Spitzer [9] was observed in 18 cases (51.4\%). Acute GvHD of grade I was observed in 6 cases (17.1\%), grade II in 10 cases $(28.6 \%)$, grade III in 8 cases $(22.9 \%)$, and grade IV in 3 cases (8.6\%). Encephalopathy occurred in two cases $(5.7 \%)$, thrombotic microangiopathy (TMA) in seven cases $(20.0 \%)$, and hepatic venous obstruction disease (hepatic VOD) in three cases (8.6\%).

In 33 patients, eight patients were dead due to transplantationrelated complications. One ALL patient relapsed, received $2^{\text {nd }}$ allogeneic 
SCT, and died because of severe hepatic VOD. Two patients rejected first SCT, and one successfully accepted $2^{\text {nd }}$ SCT, and the other was dead due to transplantation-related complication (Table 1).

Results of virus monitoring (Table 4, Figure 1): In 23 cases $(65.7 \%)$, one or more viruses were detected, and their average age was 9.4 years (from 7 months to 21 years; median of 11 years). In 12 cases, no virus was detected, and their average age was 3.1 years (from 8 months to 17 years; median of 1.5 years). HHV-6 was positive in 36 specimens (10.7\%), EBV in 34 specimens (9.9\%), and CMV in 33 specimens $(9.5 \%)$. The cumulative positive incidences were EBV of $48.6 \%$, HHV -6 of $37.1 \%$ and CMV of $28.6 \%$ (Figure 1). In the average, EBV was detected at 46.4 days, CMV at 41.1 days, and HHV-6 at 30.5 days after transplantation. In some cases, multiple viruses were detected in the same specimen at once. HHV-8 and PVB19 were not detected in

Table 3. PCR primers used for multiplex PCR and nested PCR.

\begin{tabular}{|c|c|c|}
\hline Virus & & Sequence for primers and probes \\
\hline \multirow{4}{*}{ HSV1.2 } & HSV-F & CGCATCAAGACCACCTCCTC \\
\hline & HSV-R & GTCAGCTCGTGRTTCTG \\
\hline & HSV1-probe & TGGCAACGCGGCCCAAC \\
\hline & HSV2-prpbe & CGGCGATGCGCCCCAG \\
\hline \multirow{4}{*}{ VZV } & VZV-F & TCACTACCAGTCATTTCTATCCATCTG \\
\hline & VZV-R & GAAAACCCAAACCGTTCTCGAG \\
\hline & VZV-probe & TGTCTTTCACGGAGGCAAACACGT \\
\hline & EBV-F & CTGGGCAAGGAGCTGTTTG \\
\hline \multirow[t]{2}{*}{ EBV } & EBV-R & GGCCGCTTGTAAAATTGCA \\
\hline & EBV-probe & CTCGGCTGTGGAGCAGGCTT \\
\hline \multirow{3}{*}{ CMV } & CMV-F & TCGCGCCCGAAGAGG \\
\hline & CMV-R & CGGCCGGATTGTGGATT \\
\hline & CMV-probe & CACCGACGAGGATTCCGACAACG \\
\hline \multirow{3}{*}{ HHV6 } & HHV6-F & GAAGCAGCAATCGCAACACA \\
\hline & HHV6-R & ACAACATGTAACTCGGTGTACGGT \\
\hline & HHV6-probe & AACCCGTGCGCCGCTCCC \\
\hline \multirow{3}{*}{ HHV7 } & HHV7-F & CGGAAGTCACTGGAGTAATGACAA \\
\hline & HHV7-R & CCAATCCTTCCGAAACCGAT \\
\hline & HHV7-probe & CTCGCAGATTGCTTGTTGGCCATG \\
\hline \multirow{3}{*}{ HHV8 } & HHV8-F & СCTGTCCTCTGGTCCCCAT \\
\hline & HHV8-R & ATCGTTGCCTATTTCTTTTTGCC \\
\hline & HHV8-probe & CCGGCGTCAGACATTCTCACAACC \\
\hline \multirow{4}{*}{$\mathrm{BKV} / \mathrm{JCV}$} & BKVJCV-F & GGAAAGTCTTTAGGGTCTTCTACCTTT \\
\hline & BKV-R & GATGAAGATTTATTYTGCCATGARG \\
\hline & JCV-R & GAAGACCTGTTTTGCCATGAAGA \\
\hline & BKVJCV-probe & e ATCACTGGCAAACAT \\
\hline \multirow{3}{*}{ ADV } & ADV-F & GACATGACTTTTGAGGTGGA \\
\hline & ADV-R & TCGATGACGCCGCGGTG \\
\hline & ADV-probe & CCCATGGAYGAGCCCACСCT \\
\hline \multirow{3}{*}{ PVB19 } & B19-F & GGGTTTCAAGCACAAGYAGTAAAAGA \\
\hline & B19-R & CGGYAAACTTCCTTGAAAATG \\
\hline & B19-probe & CAGTGCCCCTGTGG \\
\hline
\end{tabular}

Table 4. Results of comprehensive virus analysis after SCT.

\begin{tabular}{|c|c|c|c|c|c|c|c|c|}
\hline & Sample number & Positive sample & $\begin{array}{l}\text { Positive } \\
\text { ratio(\%) }\end{array}$ & $\begin{array}{l}\text { Positive } \\
\text { patients }\end{array}$ & $\begin{array}{c}\text { Positive patients } \\
(\%)\end{array}$ & $\begin{array}{l}\text { Mean onset day } \\
\text { (day) }\end{array}$ & $\begin{array}{l}\text { Mean peak day } \\
\quad \text { (day) }\end{array}$ & $\begin{array}{c}\text { Mean peak virus load } \\
(\mu \mathrm{gDNA})\end{array}$ \\
\hline HSV-1 & 371 & 3 & 0.8 & 3 & 8.6 & $46(0-91)$ & - & - \\
\hline HSV-2 & 350 & 1 & 0.3 & 1 & 2.9 & 47 & - & - \\
\hline VZV & 356 & 1 & 0.3 & 1 & 2.9 & 101 & - & - \\
\hline EBV & 378 & 34 & 9.9 & 17 & 48.6 & $46.4(6-110)$ & $65.4(19-110)$ & $661.1(42-2800)$ \\
\hline CMV & 379 & 33 & 9.5 & 10 & 28.6 & $41.1(0-96)$ & $52.3(7-111)$ & $15044.8(3-110000)$ \\
\hline HHV-6 & 371 & 36 & 10.7 & 13 & 37.1 & $30.5(12-96)$ & $29.1(12-96)$ & $13922.5(26-58000)$ \\
\hline HHV-7 & 367 & 6 & 1.7 & 1 & 2.9 & 8 & 8 & 4000 \\
\hline HHV-8 & 312 & 0 & 0.0 & 0 & 0 & - & - & - \\
\hline $\mathrm{BKV}$ & 321 & 3 & 0.9 & 2 & 5.7 & $70.5(54-87)$ & $68.5(50-87)$ & $42.5(35-50)$ \\
\hline $\mathrm{JCV}$ & 318 & 3 & 0.9 & 2 & 5.7 & $11.5(5-18)$ & 34 & 170 \\
\hline PVB19 & 319 & 0 & 0.0 & 0 & 0 & - & - & - \\
\hline ADV & 51 & 3 & 6.3 & 1 & 2.9 & 6 & 6 & 1800 \\
\hline
\end{tabular}

any samples. BKV and JCV were positive in two cases (pt.nos.10 and 12), respectively. ADV was detected in one case transiently (pt.nos.20).

The viral load and its significance (Figure 2): In seven patients with

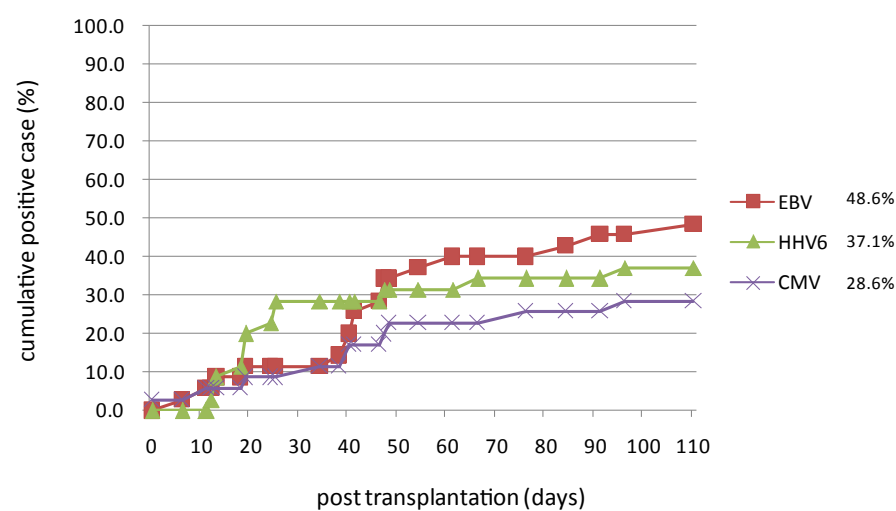

Figure 1. Cumulative incidence of viral infection after SCT.

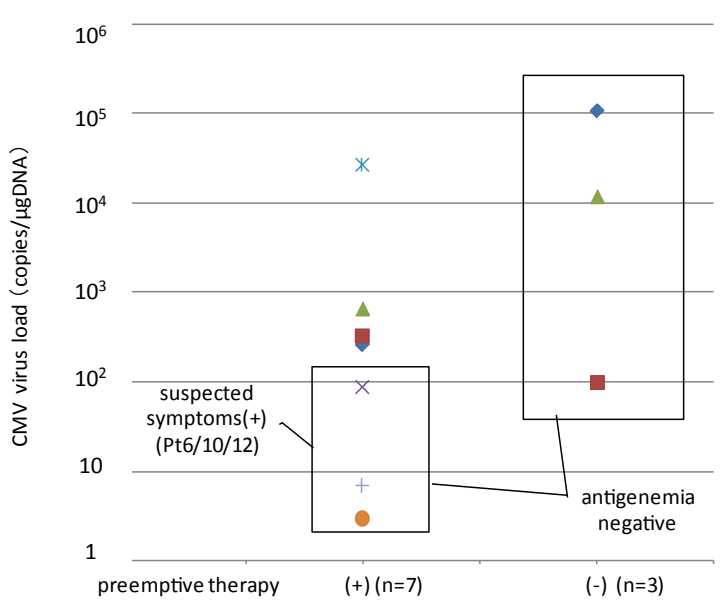

\begin{tabular}{|c|c|c|c|c|}
\hline \multirow{2}{*}{$\begin{array}{c}\text { CMV-PCR } \\
\text { (copies/MgDNA) }\end{array}$} & \multicolumn{2}{|c|}{ preemptive therapy } & \multicolumn{2}{c|}{ antigenemia } \\
\cline { 2 - 5 } & $(+)$ & $(-)$ & $\geqq 5$ & $<5$ \\
\hline$\geqq 1 \times 10^{2}$ & 4 & 3 & 4 & 3 \\
\hline$<1 \times 10^{2}$ & 3 & 0 & 0 & 3 \\
\hline
\end{tabular}
Figure 2. Preemptive CMV therapy and its relation with CMV load and CMV pp65 antigenemia. 
Table 5. Effect of viral infection before SCT on the post SCT viral infection.

\begin{tabular}{|c|c|c|c|}
\hline \multicolumn{2}{|c|}{ CMV PCR } & \multicolumn{2}{c|}{ post SCT } \\
\cline { 2 - 4 } & positive & positive & negative \\
\hline \multirow{2}{*}{ before SCT } & negative & 1 & 2 \\
\cline { 2 - 4 } & ne & 8 & 20 \\
\hline
\end{tabular}

\begin{tabular}{|l|c|c|c|}
\hline \multicolumn{2}{|c|}{ anti-CMV antibody* } & \multicolumn{2}{c|}{ post SCT } \\
\cline { 2 - 4 } & positive & negative \\
\hline \multirow{2}{*}{ before SCT } & positive & 8 & 6 \\
\cline { 2 - 4 } & negative & 1 & 10 \\
\hline
\end{tabular}

\begin{tabular}{|c|c|c|c|}
\hline \multicolumn{2}{|c|}{ EBV PCR } & \multicolumn{2}{c|}{ post SCT } \\
\cline { 2 - 4 } & positive & positive & negative \\
\hline \multirow{2}{*}{ before SCT } & negative & 7 & 2 \\
\cline { 2 - 4 } & & 8 & 14 \\
\hline
\end{tabular}

\begin{tabular}{|c|c|c|c|}
\hline \multicolumn{2}{|c|}{ HHV-6 PCR } & \multicolumn{2}{c|}{ post SCT } \\
\cline { 3 - 4 } & positive & positive & negative \\
\hline \multirow{2}{*}{ before SCT } & negative & 3 & 1 \\
\cline { 2 - 4 } & nyy & 8 & 19 \\
\hline
\end{tabular}

$*:$ Patients with antibody deficiency were excluded.

Positive EBV or anti-CMV antibody before SCT predicts EBV or CMV infection after SCT with an odds ration of 6.13 (p=0.036) and 13.3 (p=0.013) respectively.

Table 6. Risk factors for virus infection after SCT: Multivariate analysis.

\begin{tabular}{|c|c|c|c|c|c|c|c|c|c|}
\hline & \multicolumn{3}{|c|}{ HHV-6 } & \multicolumn{3}{|c|}{ EBV } & \multicolumn{3}{|c|}{ CMV } \\
\hline & odds ratio & $95 \%$ interval & P-value & odds ratio & $95 \%$ interval & P-value & odds ratio & $95 \%$ interval & P-value \\
\hline non-malignacy & 0.02 & $0.00-1.06$ & 0.05 & 1.10 & $0.14-8.87$ & 0.93 & 0.52 & $0.03-9.51$ & 0.66 \\
\hline age $\geqq 9$-year-old & 1.94 & $0.29-12.7$ & 0.49 & 2.79 & $0.45-17.4$ & 0.27 & 1.36 & $0.16-11.8$ & 0.78 \\
\hline unrelated cord blood & 1.72 & $0.17-17.5$ & 0.65 & 0.30 & $0.04-2.14$ & 0.23 & 0.33 & $0.02-6.23$ & 0.46 \\
\hline ATG & 40.03 & $1.21-1320$ & $0.04 *$ & 2.58 & $0.19-34.6$ & 0.47 & 6.82 & $0.14-338$ & 0.34 \\
\hline RIC & 8.65 & $0.43-173$ & 0.16 & 0.40 & $0.04-3.67$ & 0.42 & 0.02 & $0.00-1.79$ & 0.09 \\
\hline aGVHD III-IV & 0.15 & $0.01-1.63$ & 0.12 & 6.25 & $0.74-52.7$ & 0.09 & 10.47 & $0.78-139$ & 0.08 \\
\hline
\end{tabular}

Multivariate analysis showed that GVHD( $\geqq 3$ ) was the most significant factor for CMV (odds ratio $=10.47$ ) and EBV (odds ratio $=6.25)$ and $\mathrm{ATG}$ for HHV-6 (odds ratio $=40.03$ ).

CMV load of $1 \times 10^{2}$ copies/ $\mu$ g DNA or more, CMV pp65 antigenemia was positive (more than 5 positive cells out of 50,000 cells) in four cases who received anti-CMV agents (Figure 2). Whereas asymptomatic three cases (pt.nos.7, 13 and 34) with CMV pp65 antigenemia negative (less than 5 positive cells out of 50,000 cells) turned negative for CMV without anti-CMV agent even if CMV load was transiently $1 \times 10^{2}$ copies/ $\mu$ g DNA or more after the continued inspection. Three cases (pt.nos. 6, 10 and 12) with CMV load of $1 \times 10^{2}$ copies/ $\mu g$ DNA or less received anti-CMV agents because of suspected symptoms.

Other than CMV, viral load became decreased or negative along with the recovery of immune function without any intervention.

EBV load was less than $1 \times 10^{4}$ copies/ $\mu$ g DNA in all cases, and no one developed lymphoproliferative disease.

As for HHV-6, more than $1 \times 10^{4}$ copies/ $\mu \mathrm{g}$ DNA of HHV-6 was detected in five cases.

One case (pt.nos. 8) was transplanted at 11-month-old and HHV-6 was detected as a primary infection at $96^{\text {th }}$ day after transplantation. In other four (pt.nos. 11, 19, 22 and 34: aged between 5 and 6 years), the peak of HHV-6 load was between 13 and 19 days after transplantation as a reactivation. In the SCT of adult patients, significance of HHV6 infection is being emphasized, especially as a causative pathogen of encephalitis. Clinical significance of HHV-6 infection in the pediatric SCT is still controversial $[10,11]$.

Association with virus infection status before the transplant (Table 5): We examined 31 cases in which viral PCR analysis was performed within one month before transplantation to find out the relation with virus reactivation after SCT.

CMV was detected in one out of three CMV positive for PCR (33.3\%) and in 8 out of 28 cases (28.6\%) in which CMV was negative for PCR before transplantation. In 14 cases with positive anti-CMV antibody, CMV was detected in 8 cases $(57.1 \%)$ after transplantation. Out of 11 cases in which anti-CMV antibody was negative before transplantation, CMV was detected in only one case (9.1\%).

EBV was detected in 7 out of 9 cases (77.8\%) in which EBV was positive for PCR before transplantation, and HHV-6 was detected in 3 out of 4 cases (75\%) in which HHV-6 was positive for PCR before transplantation. On the contrary, EBV was detected in 8 out of 22 cases (36.4\%) in which EBV was negative for PCR before transplantation. HHV- 6 was detected in 8 out of 27 cases (29.6\%) in which HHV-6 was negative for PCR before transplantation.

Risk factor for virus infection (Table 6): To find out the risk factors for viral infection after SCT, we performed multivariate analysis. Only ATG administration and HHV-6 infection (odds ratio 40.03, $\mathrm{p}=0.04$ ) showed a statistically significant relevance. GvHD IIIIV was related with CMV (odds ratio 6.25, $\mathrm{p}=0.09$ ) and EBV infection (odds ratio 10.47, $\mathrm{p}=0.08$ ), and RIC was related with HHV-6 infection (odds ratio $8.65, \mathrm{p}=0.16$ ), although not statistically significant (Table 4). TMA was associated with CMV infection (odds ratio 7.91, $\mathrm{p}=0.09$ ), but not with EBV (odds ratio 0.46, $\mathrm{p}=0.52$ ) or HHV-6 infection (odds ratio $1.36, \mathrm{p}=0.74$ ), which is different from the report with adult SCT [12]. Unrelated cord blood was not related with HHV-6 infection.

Clinical impact of virus monitoring on TRM in pediatric SCT: To investigate the clinical impact of virus monitoring on the outcome of SCT, we compared TRM in 2004-2009 cohort with TRM in 19982004 cohort. The patient profiles of 1998-2004 cohort were presented precisely in Table 2. SCT procedure and supportive care were the same as described above in Methods. Engraftment syndrome was not recorded in this cohort because the concept and criteria of ES were not accepted in this period. The content of background diseases and conditioning regimens were comparable between two cohorts. As 
shown in Figure 3, TRM in 2004-2009 cohort was 0.208 and TRM in 1998-2004 was 0.301, and the introduction of virus monitoring improved TRM, although not statistically significant.

\section{Discussion}

It has been reported that viruses were detected from the blood in 48 to $63 \%$ of the pediatric patients until 100 days after HSCT $[13,14]$. HHV6 has been reported to be detected two to four weeks earlier than CMV, and the reported frequency has varied from several to $70 \%[13,15-20]$. The frequency to detect CMV and EBV has been reported to be $11-28 \%$ and $24-48 \%$, respectively $[13,21,22]$. In our observation, the frequency of virus detection was almost the same as previously reported. However, it was significantly lower in infants in which primary immunodeficient patients were dominantly involved. The average age of virus-positive group was 9.4 years (median of 11 years), and that of virus-negative group was 3.1 years (median 0.5 years). Especially, in the 15 patients of one year old or younger (infants group), EBV was detected in only 4 cases (26.7\%), CMV in one (6.7\%), and HHV-6 in three (20.0\%). No virus was detected in 9 patients $(60.0 \%)$. In the infants group, majority of patients had not encountered viral infections before SCT. Most of the immunnodeficient patients had been managed to be protected from infection since they were diagnosed. These patients are at risk for primary infection from the transfusions. It has been reported that CMV negative donor is preferable to avoid CMV infection in these patients [23]. Furthermore, transfusion-associated CMV is reported to be prevented by using the leukocyte-filtered blood products [24]. In our institute, CMV negative donor could not be supplied in any of the cases, but all of blood products were leukocyte-filtered. In the older children, they are already infected and reactivation of virus often becomes a clinical problem as in the adult case under the secondary immunodeficient condition after SCT [20].

We re-evaluated the correlation of CMV viral load and CMV pp65 antigenemia level, and its association with the prognosis in the pediatric SCT [25-27].

Three cases (pt.nos.6, 10 and 12) with CMV load of $1 \times 10^{2}$ copies/ $\mu \mathrm{g}$ DNA or less received anti-CMV agents because of suspected symptoms, although their CMV pp65 antigenemia were negative. Three in 7 cases with CMV load of $1 \times 10^{2}$ copies/ $\mu$ D DNA and more were CMV pp65 antigenemia negative. All of CMV pp65 antigenemia positive patients showed CMV load of $1 \times 10^{2}$ copies/ $\mu \mathrm{g}$ DNA and more. As previously reported, sensitivity of CMV pp65 antigenemia is unstable and unreliable when leukocyte number is low during the early phase of SCT [26]. These results confirm that PCR testing is more

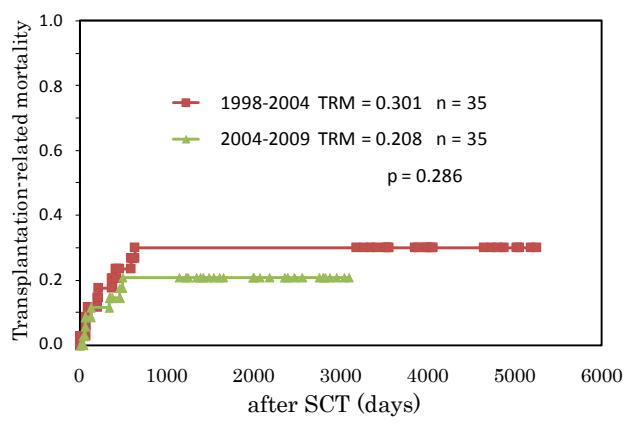

Transplantation-related mortality (TRM) of 2004-2009 cohort was compared with that of a historical control cohort between 1998 and 2004 when virus monitoring was not introduced.

Figure 3. Clinical impact of virus monitoring on TRM in pediatric SCT. sensitive than antigenemia and is more suitable for monitoring after SCT.

To start preemptive intervention, the level of $1 \times 10^{2}$ copies/ $\mu \mathrm{g}$ DNA and more or CMV pp65 antigenemia of 5 and more out of 50,000 cells seemed to be reasonable practically in our experience as suggested before [27]. However, preemptive therapy should be started for the patients with suspected symptoms, even if viral DNA load is lower than cutoff level.

It has been reported that more than $1 \times 10^{2.5}$ copies/ $\mu \mathrm{g}$ DNA of EBV load is a sign of viral reactivation, and more than $1 \times 10^{4}$ copies $/ \mu \mathrm{g}$ DNA is a risk factor for EBV lymphoproliferative disease (EBV-LPD) [28]. In this study, four cases showed $1 \times 10^{2.5}$ copies $/ \mu$ g DNA or more of EBV load, but not exceed $1 \times 10^{4}$ copies/ $\mu \mathrm{g}$ DNA and all of them resolved spontaneously. Clinical point of view, EBV load of $1 \times 10^{4}$ copies/ug DNA or more seems reasonable as a cutoff for preemptive intervention of EBV-LPD such as anti-CD20 antibody administration.

It has been reported that the infection history of both of donor and recipient is associated with viral infection and reactivation after SCT $[13,17]$.

In the PCR positive case of EBV and CMV before transplant, the same virus was more likely to be detected after transplant (Table 5). For CMV infection, the antibody test of recipients before transplant was also important. Positivity of antibody for CMV was a risk of reactivation even if PCR testing before transplantation was negative. In some cases (pt.nos. 11, 22 and 30), CMV was positive in stool and pharynx, but negative in blood before SCT, and then CMV was detected in the blood after SCT. In cases of antibody-production deficiency, direct detection of virus before SCT is an only method to find out the previous or insidious infection of CMV. In the patients with immunodeficiency, viruses are often detected persistently even without clinical symptoms. The evaluation of antibody and virus before transplant is also useful and important as well as early preemptive detection of virus by the regular monitoring after SCT.

UR-BM, HLA mismatch, URCB, MAC, T-cell-depleted transplant, ATG, steroid, and GvHD have been reported to be risk factors for virus infection and reactivation [13-16,18,25]. In our study, HHV-6 infection was not related with unrelated cord blood contrary to the report from adults SCT [18]. This may be due to the fact that most of unrelated cord blood was transplanted to the patients under two years old, who might not be infected with HHV-6 yet. A statistically significant relevance was found between ATG administration and HHV-6 infection. However, CMV and EBV infection were not related with ATG. CMV and EBV infection were related with acute GvHDIII-IV, although not statistically significant. This may be interpreted that short-term intensive immunosuppression is related with HHV-6 infection/reactivation, while long-term immunosuppression combined with inflammation is prerequisite for CMV and EBV infection/reactivation.

Finally, in order to investigate the clinical impact of sequential virus monitoring, we compared TRM of this cohort with that of historical control in which virus monitoring was not introduced.

TRM in 2004-2009 cohort was 0.208 and TRM in 1998-2004 was 0.301 , and the introduction of virus monitoring improved TRM, although not significantly (Figure 3).

GvHD II-IV and GvHD III-IV were 60\% (21/35) and 31.4\% (11/35) in 2004-2011 cohort and 40\% (14/35) and 20\% (7/35) in 19982004, which indicates that 2004-2011 cohort contained more high risk 
patients than 1988-2004 cohort. However, we have to be cautious in the interpretation of this result because the number of patients in both cohorts was small and the profiles of both patients and conditioning regimen were not the same. Furthermore, TRM is dependent on not only virus infection but also other SCT-related risk factors such as donor source, conditioning regimen, HLA-disparity, treatment history, and so on.

Although, the introduction of virus monitoring might not have statistically significant impact of the improvement of TRM, future development of novel anti-virus therapy such as virus-specific adoptive cell therapy and anti-virus medicine will improve the clinical consequences ultimately in combination with early detection of virus infection by sequential virus monitoring. Actually, it has been reported that pre-empiric anti-CD20 administration after early detection of EBV is useful for the prevention of development of EBV-LPD after SCT $[29,30]$.

Our observation seems instructive and further examination is necessary to resolve clinical problems with viral infection in pediatric SCT.

\section{Acknowledgement}

This work was partly supported by Grant-in-Aid for Scientific Research (KAKENHI) (grant nos.: 16659273, 20591246, 24591541), which was received by M.N.

\section{Author contributions}

Nagasawa M contributed to study conception and design, data analysis, and writing of a paper. Mitsuiki $\mathrm{N}$ contributed to data analysis and writing of a paper. Endo A, Aoki Y, Ono T, Isoda T, Takagi M, and Kajiwara M contributed to data collection. Shimizu N contributed to virus analysis and Morio $\mathrm{T}$ contributed to final approval of a paper.

\section{Institutional review board statement}

This study was reviewed and approved by the Tokyo Medical and Dental University Hospital Institutional Review Board.

\section{Informed consent statement}

All study participants, or their legal guardian, provided informed consent prior to study enrollment.

\section{Conflict-of-interest statement}

The authors have no conflict-of-interest to declare.

\section{References}

1. Sullivan KM, Dykewicz CA, Longworth DL, Boeckh M, Baden LR, et al. (2001) Preventing opportunistic infections after hematopoietic stem cell transplantation: the Centers for Disease Control and Prevention, Infectious Diseases Society of America, and American Society for Blood and Marrow Transplantation Practice Guidelines and beyond. Hematology Am Soc Hematol Educ Program 392-421. [Crossref]

2. Junghanss C, Boeckh M, Carter RA, Sandmaier BM, Maris MB, et al. (2002) Incidence and outcome of cytomegalovirus infections following nonmyeloablative compared with myeloablative allogeneic stem cell transplantation, a matched control study. Blood 99: 1978-1985. [Crossref]

3. Li CR, Greenberg PD, Gilbert MJ, Goodrich JM, Riddell SR. (1994) Recovery of HLA-restricted cytomegalovirus (CMV)-specific T-cell responses after allogeneic bone marrow transplant: correlation with CMV disease and effect of ganciclovir prophylaxis. Blood 83: 1971-1979. [Crossref]

4. Winston DJ, Ho WG, Bartoni K, Du Mond C, Ebeling DF, et al. (1993) Ganciclovir prophylaxis of cytomegalovirus infection and disease in allogeneic bone marrow transplant recipients. Results of a placebo-controlled, double-blind trial. Ann Intern
Med 118: 179-184. [Crossref]

5. Goodrich JM, Mori M, Gleaves CA, Du Mond C, Cays M, et al. (1991) Early treatment with ganciclovir to prevent cytomegalovirus disease after allogeneic bone marrow transplantation. N Engl J Med 325: 1601-1607. [Crossref]

6. Heid CA, Stevens J, Livak KJ, Williams PM (1996) Real time quantitative PCR Genome Res 6: 986-994. [Crossref]

7. Ririe KM, Rasmussen RP, Wittwer CT (1997) Product differentiation by analysis of DNA melting curves during the polymerase chain reaction. Anal Biochem 245: 154160. [Crossref]

8. Sugita S, Shimizu N, Watanabe K, Mizukami M, Morio T, et al. (2008) Use of multiplex PCR and real-time PCR to detect human herpes virus genome in ocular fluids of patients with uveitis. Br J Ophthalmol 92: 928-932. [Crossref]

9. Spitzer TR. (2001) Engraftment syndrome following hematopoietic stem cell transplantation. Bone marrow transplan 27: 893-898. [Crossref]

10. Verhoeven DH, Claas EC, Jol-van der Zijde CM, Thijssen JC, Lankester AC, et al. (2015) Reactivation of Human Herpes Virus-6 After Pediatric Stem Cell Transplantation: Risk Factors, Onset, Clinical Symptoms and Association with Severity of Acute GraftVersus-Host Disease. Pediatr Infect Dis J 34: 1118-11127. [Crossref]

11. Violago L, Jin Z, Bhatia M, Rustia E, Kung AL, et al. (2015) Human herpesvirus-6 viremia is not associated with poor clinical outcomes in children following allogeneic hematopoietic cell transplantation. Pediatr transplant 19: 737-744. [Crossref]

12. Takatsuka H, Wakae T, Mori A, Okada M, Fujimori Y, et al. (2003) Endothelial damage caused by cytomegalovirus and human herpesvirus-6. Bone marrow transplant 31: 475-479. [Crossref]

13. Schonberger S, Meisel R, Adams O, Pufal Y, Laws HJ, et al. (2010) Prospective, comprehensive, and effective viral monitoring in children undergoing allogeneic hematopoietic stem cell transplantation. Biol Blood Marrow Transplant 16: 1428-1435. [Crossref]

14. Verdeguer A, de Heredia CD, Gonzalez M, Martinez AM, Fernandez-Navarro JM, et al. (2011) Observational prospective study of viral infections in children undergoing allogeneic hematopoietic cell transplantation: a 3-year GETMON experience. Bone marrow transplant 46: 119-124. [Crossref]

15. Boeckh M, Erard V, Zerr D, Englund J (2005) Emerging viral infections after hematopoietic cell transplantation. Pediatr transplant. 9 Suppl 7: 48-54. [Crossref]

16. Chatzidimitriou D, Gavriilaki E, Sakellari I, Diza E (2010) Hematopoietic cell transplantation and emerging viral infections. J Med Virol 82: 528-538. [Crossref]

17. Fischer SA. (2008) Emerging viruses in transplantation: there is more to infection after transplant than CMV and EBV. Transplantation 86: 1327-1339. [Crossref]

18. Sashihara J, Tanaka-Taya K, Tanaka S, Amo K, Miyagawa H, et al. (2002) High incidence of human herpesvirus 6 infection with a high viral load in cord blood stem cell transplant recipients. Blood 100: 2005-2011. [Crossref]

19. Savolainen H, Lautenschlager I, Piiparinen H, Saarinen-Pihkala U, Hovi L, et al. (2005) Human herpesvirus-6 and -7 in pediatric stem cell transplantation. Pediatr Blood Cancer 45: 820-825. [Crossref]

20. Yoshikawa T, Suga S, Asano Y, Nakashima T, Yazaki T, et al. (1991) Human herpesvirus-6 infection in bone marrow transplantation. Blood 78: 1381-1384. [Crossref]

21. Cohen J, Gandhi M, Naik P, Cubitt D, Rao K, et al. (2005) Increased incidence of EBVrelated disease following paediatric stem cell transplantation with reduced-intensity conditioning. Br J haematol 129: 229-239. [Crossref]

22. Wada K, Kubota N, Ito Y, Yagasaki H, Kato K, et al. (2007) Simultaneous quantification of Epstein-Barr virus, cytomegalovirus, and human herpesvirus 6 DNA in samples from transplant recipients by multiplex real-time PCR assay. J Clin Microbiol 45: 1426-1432. [Crossref]

23. Nichols WG, Corey L, Gooley T, Davis C, Boeckh M (2002) High risk of death due to bacterial and fungal infection among cytomegalovirus (CMV)-seronegative recipients of stem cell transplants from seropositive donors: evidence for indirect effects of primary CMV infection. $J$ Infect Dis 185: 273-282. [Crossref]

24. Bowden RA, Slichter SJ, Sayers M, Weisdorf D, Cays M, et al. (1995) A comparison of filtered leukocyte-reduced and cytomegalovirus (CMV) seronegative blood products for the prevention of transfusion-associated CMV infection after marrow transplant Blood 86: 3598-3603. [Crossref]

25. Asano-Mori Y, Oshima K, Sakata-Yanagimoto M, Nakagawa M, Kandabashi K, et al. (2005) High-grade cytomegalovirus antigenemia after hematopoietic stem cell 
transplantation. Bone marrow transplant 36: 813-819. [Crossref]

26. Boeckh M, Bowden RA, Goodrich JM, Pettinger M, Meyers JD (1992) Cytomegalovirus antigen detection in peripheral blood leukocytes after allogeneic marrow transplantation. Blood 80: 1358-1364. [Crossref]

27. Kanda Y, Yamashita T, Mori T, Ito T, Tajika K, et al. (2010) A randomized controlled trial of plasma real-time PCR and antigenemia assay for monitoring CMV infection after unrelated BMT. Bone marrow transplant 45: 1325-1332. [Crossref]

28. Hoshino Y, Kimura H, Tanaka N, Tsuge I, Kudo K, et al. (2001) Prospective monitoring of the Epstein-Barr virus DNA by a real-time quantitative polymerase chain reaction after allogenic stem cell transplantation. Br J haematol 115: 105-111. [Crossref]

29. van Esser JW, Niesters HG, van der Holt B, Meijer E, Osterhaus AD, et al. (2002) Prevention of Epstein-Barr virus-lymphoproliferative disease by molecular monitoring and preemptive rituximab in high-risk patients after allogeneic stem cell transplantation. Blood 99: 4364-4369. [Crossref]

30. Rouce RH, Louis CU, Heslop HE (2009) Epstein-barr virus related lymphoproliferations after stem cell transplantation. Curr Opin Hematol 21: 476-481 [Crossref]

Copyright: (O2016 Nagasawa M. This is an open-access article distributed under the terms of the Creative Commons Attribution License, which permits unrestricted use, distribution, and reproduction in any medium, provided the original author and source are credited. 